\title{
1.17 SKIN AND SOFT TISSUE INFECTIONS
}

Cellulitis is a bacterial infection of the skin and subcutaneous tissues. It is a common, potentially serious medical condition that can result in significant morbidity and hospitalization. Annually, more than 620,000 hospital discharges occur with skin and soft tissue infection as the primary diagnosis. ${ }^{1}$ Potential complications include abscess formation. Hospitalists can lead efforts to standardize care delivery, promote antibiotic stewardship, improve discharge planning, and promptly identify and address severe cases of cellulitis to minimize complications and improve patient outcomes.

\section{KNOWLEDGE}

Hospitalists should be able to:

- Describe the clinical presentation of cellulitis and distinguish between routine and complicated cellulitis.

- Differentiate cellulitis from chronic venous stasis changes and other mimicking skin conditions and discuss the accuracy of common signs and symptoms in patients with suspected cellulitis.

- Describe the tests used to evaluate cellulitis.

- Discuss possible causative organisms on the basis of classic associations with characteristic host exposures.

- Describe factors associated with an increased risk of worsening disease severity and complications.

- Recognize indications for early specialty consultation in patients with complications, misdiagnosis, or lack of response to therapy.

- Differentiate empiric antibiotic regimens for uncomplicated and complicated types of cellulitis.

- Explain indications for outpatient treatment and need for hospital admission.

- Explain goals for hospital discharge, including specific measures of clinical stability for safe care transition.

\section{SKILLS}

Hospitalists should be able to:

- Elicit a focused medical history to identify precipitating causes of cellulitis and comorbid conditions that may affect clinical management.

- Assess patients with cellulitis in a timely manner and manage or comanage patients with the primary requesting service.

- Accurately identify routine cellulitis borders and signs of complications, which may include crepitus and abscess.

- Recommend an appropriate, cost-effective initial diagnostic evaluation of cellulitis, including laboratory and radiologic studies.

- Initiate empiric antibiotic treatment of cellulitis on the basis of host exposures, predisposing underlying systemic illness, history and physical examination findings, presumptive bacterial pathogens, and evidence-based recommendations.
- Treat coexisting fungal infection, edema, and other conditions that may exacerbate cellulitis.

- Formulate a subsequent treatment plan that includes narrowing antibiotic therapies on the basis of available culture data and the patient's response to treatment.

- Determine appropriate timing for transition from intravenous to oral therapy and duration of antibiotic treatment.

- Initiate preventive measures for minimizing risk of recurrent cellulitis.

- Communicate with patients and families to explain the history and prognosis of cellulitis.

- Communicate with patients and families to explain tests and procedures and their indications and to obtain informed consent.

- Communicate with patients and families to explain the goals of care, discharge instructions, and management after hospital discharge to ensure safe follow-up and transitions of care.

- Facilitate discharge planning early during hospitalization.

- Document the treatment plan and provide clear discharge instructions for postdischarge clinicians.

\section{ATTITUDES}

Hospitalists should be able to:

- Employ a multidisciplinary approach in the care of patients with cellulitis that begins at admission and continues through all care transitions.

- Follow evidence-based recommendations to guide diagnosis, monitoring, and treatment of cellulitis.

- Consider cost-effectiveness (including formulary availability), risk of potential adverse effects, and ease of conversion to outpatient treatment when choosing among therapeutic options.

\section{SYSTEM ORGANIZATIONS AND IMPROVEMENT}

To improve efficiency and quality within their organizations, hospitalists should:

- Lead, coordinate, and/or participate in multidisciplinary initiatives, which may include collaborative efforts with infectious disease physicians, to promote patient safety and optimize cost-effective diagnostic and management strategies for patients with cellulitis.

- Implement systems to ensure hospital-wide adherence to national standards and document those measures as specified by recognized organizations.

\section{Reference}

1. Agency for Healthcare Research and Quality. Healthcare Cost and Utilization Project. U.S. Department of Health \& Human Services. Available at: http:// hcupnet.ahrq.gov/. Accessed July 2015. 